\title{
Trial-to-trial sequential dependencies in a social and non-social Simon task
}

\author{
Roman Liepelt $\cdot$ Dorit Wenke $\cdot$ Rico Fischer . \\ Wolfgang Prinz
}

Received: 5 March 2010/Accepted: 1 November 2010

(C) Springer-Verlag 2010

\begin{abstract}
Recent research has shown that joint-action effects in a social Simon task provide a good index of action co-representation. The present study aimed to specify the mechanisms underlying joint action by considering trial-to-trial transitions. Using non-social stimuli, we assigned a Simon task to two participants. Each was responsible for only one of two possible responses. This task was performed alone (Individual go/nogo task) and in cooperation with another person who was sitting alongside (Joint go/nogo task). As a further control task, we added a Standard Simon task. Replicating previous findings (Sebanz et al. in Cognition 88:B11-B21, 2003), we found no spatial compatibility effect in the Individual go/nogo task but we did find one in the Joint go/nogo task. A more detailed analysis showed that a sequential modulation of
\end{abstract}

The authors wish to thank Julia Haupt for help with data acquisition, Bernhard Hommel, Birte Forstmann and two anonymous reviewers for helpful comments on a previous version of the manuscript.

R. Liepelt · D. Wenke - W. Prinz

Department of Psychology, Max Planck Institute for Human

Cognitive and Brain Sciences, Leipzig, Germany

R. Liepelt $(\square)$

Department of Psychology,

Junior Group "Neurocognition of Joint Action",

Westfälische Wilhelms-University, Fliednerstrasse 21,

48149 Münster, Germany

e-mail: roman.liepelt@uni-muenster.de; liepelt@cbs.mpg.de

R. Fischer

Department of Psychology, Technische Universität Dresden, Dresden, Germany

D. Wenke

Department of Experimental, Clinical and Health Psychology,

Ghent University, Ghent, Belgium the Simon effect was present in both the Joint and the Individual go/nogo tasks. We found reliable Simon effects in trials following Simon compatible trials not only in the Joint go/nogo task but also to a somewhat smaller extent in the Individual go/nogo task. For both these go/nogo tasks, sequential modulation effects were stronger for nogo/go transitions than for go/go transitions. This suggests that low-level feature binding and repetition mechanisms contribute to the social Simon effect related to the specific requirement not to respond on nogo trials.

\section{Introduction}

Most actions we perform in daily life are carried out in a social context with others. Joint action has been suggested to require the sharing of representations with others, anticipation of their behavior, and coordination of one's own actions with those of others (Sebanz, Bekkering, \& Knoblich, 2006). Some recent studies indicate that another person's action may also be co-represented within an individual's own motor system when the other person performs a complementary action in response to a different task aspect in a shared task context (Newman-Norlund, van Schie, van Zuijlen, \& Bekkering, 2007).

In task-sharing experiments, participants typically respond to different stimuli within the same task and hence perform complementary go/nogo tasks. Joint task performance is typically compared either to an Individual go/ nogo task in which the same go/nogo task is performed alone or to a condition in which complementary actions are attributed to an unintentional agent (such as the test computer). Although it is possible for participants to ignore one another in the Joint go/nogo task in such settings, findings from several studies indicate that the co-actor's actions are 
co-represented within the participant's own motor system. Co-representation suggests vicarious response activation upon seeing the other's stimulus (e.g., Ramnani \& Miall, 2004; van Schie, Mars, Coles, \& Bekkering, 2004).

Many demonstrations of action co-representation in task-sharing contexts have used the Simon task (Simon, 1990; Simon, Hinrichs, \& Craft, 1970). The basic Simon effect occurs if spatially defined responses, such as left and right key presses, are made to non-spatial form attributes, like for example, a diamond or square that is presented on the left or right side of a monitor. Although stimulus location is completely irrelevant in such a task, spatial stimulus-response (S-R) compatibility consistently produces better performance than incompatibility (De Jong, Liang, \& Lauber, 1994; Lu \& Proctor, 1995; Simon \& Rudell, 1967). Following the idea of common coding between perception, intention, and action (Liepelt, von Cramon, \& Brass, 2008; Prinz, 1997), the theory of event coding (Hommel, Müsseler, Ascherschleben, \& Prinz, 2001) explains the Simon effect as a product of a binding process. Binding occurs between the relevant stimulus feature (e.g., diamond or square) and the corresponding response feature (left or right), with the latter controlling the motor program. When a stimulus is presented on the left or right side of a display, its spatial code matches the code of one of the response features that is also involved in one of the bindings to the stimulus features and will lead to priming of this binding. This results in facilitation of spatially corresponding $\mathrm{S}-\mathrm{R}$ pairs and response competition for non-corresponding pairs.

The Simon effect is usually not observed when an individual participant performs a simple go/nogo response (Ansorge \& Wühr, 2004), suggesting that only responses that are spatially coded (e.g., as left vs. right) can be automatically activated by spatially corresponding stimuli. Using social pointing stimuli, Sebanz, Knoblich, and Prinz (2003) tested if the Simon effect reappeared in a go/nogo task when another individual performed the complementary go/nogo task by responding to the other target (see also Tsai, Kuo, Jing, Hung, \& Tzeng, 2006). Sebanz et al. (2003) compared three types of Simon tasks. In the Individual go/nogo task, participants performed simple go/ nogo responses that required a response to one stimulus attribute (e.g., a red ring on a left- or right-pointing finger) and the withholding of the responses to the other stimulus attribute (e.g., a green ring on a left- or right-pointing finger). In the Joint go/nogo task, two participants sat alongside each other and performed complementary go/ nogo tasks (e.g., one participant responded to red, the other to green). Finally, they included a standard Simon control task in which individual participants reacted with twochoice responses to both stimulus attributes. They found a Simon effect in the standard Simon task, but not in the
Individual go/nogo task. However, the Simon effect reappeared in the Joint go/nogo task. Based on these findings, the authors concluded that both actors form shared representations in joint task contexts, such that the action alternative of the other person is also represented (corepresentation).

More recently, evidence of co-representation has also been shown when the social pointing stimuli were replaced with abstract non-social stimuli (e.g., Tsai et al., 2006). In line with this finding, Sebanz et al. proposed that the social Simon effect was established by the spatial (left-right) coding elicited by the role of a second co-actor. More broadly, they argued that the spatial stimulus attributes induce automatic response activation. When the irrelevant spatial information conflicts with the spatial response to be given, response conflict arises (Sebanz et al., 2003; Wallace, 1971) - analogous to the Simon effect observed with the standard two-choice Simon task. This implies that, although coordination is not explicitly required in most joint-action paradigms, task sharing seems to involve coactivation of the other person's S-R mappings (Sebanz, Knoblich, \& Prinz, 2005). The re-appearance of the Simon effect in the Joint go/nogo task is typically explained by the assumption that the co-actor's actions are represented "just like one's own", rendering the Joint go/nogo task more similar to the standard two-choice version of the Simon task than to the Individual go/nogo task. Thus, co-representation will only work when another acting person is present (Joint go/nogo task) but not in the Individual go/ nogo task where no other person is present. In the Individual go/nogo task, there is no need for co-representation. According to this view, task representation and performance in a Joint go/nogo task is functionally the same as in a standard Simon task, but different from an Individual go/ nogo task (Sebanz et al., 2003; Tsai \& Brass, 2007; van Schie et al., 2004).

Given the findings of a social Simon effect in the Joint go/nogo task, this view seems plausible, but may be too simplified. This assumption overlooks possible functional similarities between the Individual go/nogo and the Joint go/nogo tasks. These tasks have a lot in common. For example, both tasks require participants to respond to only one stimulus attribute (e.g., red) and withhold the response to the other stimulus attribute (e.g., green), a requirement that changes from trial to trial. We assume that the Individual go/nogo and Joint go/nogo tasks are based on the same cognitive mechanisms. If this is true, one should not only analyze the commonalities of the two go/nogo tasks with respect to the overall Simon effect. One should also look for commonalities beyond this measurement, such as trial-to-trial sequential effects (e.g., dependence on previous Simon compatibility and/or dependence on previous go/nogo requirements), and RT distributions. These 
measurements may allow the detection of more fine-grained functional similarities and differences between the Individual and the Joint go/nogo tasks.

The present study aimed to study the underlying mechanisms of the social Simon effect attributed to action co-representation in more detail. In contrast to previous studies that focused on the interaction between setting (Joint, Standard Simon task) and the Simon effect, we investigated the trial-to-trial sequential dependencies (e.g., Gratton, Coles, \& Donchin, 1992) of the Simon effect. We focus on the comparison between Individual and Joint Simon effects. A typical finding when investigating Simon compatibility effects, for example, is that the size of the Simon effect critically depends on the characteristics of the preceding trial. In particular, the sequential modulation effect reflects the finding that Simon effects in a current trial $N$ are smaller following incompatible trials in $N-1$ compared to those following compatible trials in $N-1$ (Akçay \& Hazeltine, 2007; Fischer, Dreisbach, \& Goschke, 2008; Hommel, Proctor, \& Vu, 2004; Notebaert, Soetens, \& Melis, 2001; Stürmer, Leuthold, Soetens, Schröter, \& Sommer, 2002; Wühr \& Ansorge, 2005).

In binary choice tasks, such sequential modulation effects have often been explained in terms of bottom-up driven S-R feature repetitions and/or feature bindings (Hommel, 1998; Hommel et al., 2001, 2004; Nieuwenhuis, Stins, Posthuma, Polderman, Boomsma, \& De Geus, 2006; Notebaert et al., 2001; Wendt, Kluwe, \& Peters, 2006). ${ }^{1}$ If a stimulus and the response to the stimulus co-occur in time, their features related to task-relevant stimulus or response dimensions are spontaneously integrated into a common event file comprising both stimulus- and response-related feature information such as form and position (Hommel et al., 2004). This typically results in reaction time (RT) benefits for complete $\mathrm{S}-\mathrm{R}$ repetitions/alternations, which occur in compatible-compatible or incompatible-incompatible trial conversions from trial $N-1$ to $N$. At the same time, RT costs are found for partial S-R repetitions/alternations, which occur in compatible-incompatible or incompatible-compatible trial conversions from trial $N-1$ to $N$ (e.g., Hommel et al., 2004). Therefore, at least in a standard Simon task, S-R repetition and feature binding mechanisms seem to play an important role in determining the occurrence and the size of the Simon effect observed.

To our knowledge, however, such low-level mechanisms have so far been neglected in the often-used social variant of the Simon task. The issue of whether low-level mechanisms also contribute to the social Simon effect is an

\footnotetext{
${ }^{1}$ For an alternative explanation of sequential modulation effects holding that reduced interference after incompatible trials is due to increased cognitive control, see Botvinick, Braver, Barch, Carter, \& Cohen (2001); Gratton et al. (1992); Stürmer et al. (2002); Winkel et al. (2009); Wühr (2005); Wühr \& Ansorge (2005).
}

important question. This is because trial-to-trial sequences have a different quality in Joint and Individual go/nogo tasks (as compared to a standard Simon task), in which each person responds to one stimulus only. Transitions from trial $N-1$ to $N$ not only contain repetitions/alternations of particular stimulus features but also information as to whether a response to the previous stimulus was necessary (go trial) or not (nogo trial). Therefore, in a social Simon task, common event files will not only contain form and location related features as in a conventional Simon task, but also information about potentially inhibited locations in nogo trials (e.g., inhibitory response tags) that might affect processing in subsequent trials. This raises the question as to how binding affects performance in Individual and Joint go/nogo tasks.

Event-file integration predicts specific effects for different trial transitions, such as in go and nogo trials (see Fig. 2). Let us consider, for example, nogo/go trial transitions in which the other person (e.g., left side) responds in $N-1$ (nogo trial) and the actor (e.g., right side) responds in trial $N$ (go trial). If the person on the left responds in an incompatible trial, the stimulus is presented on the right side. The location might trigger a response activation for the person on the right side that needs to be inhibited because it is a nogo trial for this person. Therefore, the location in which the stimulus appears might be associated or "tagged" with inhibition. Such an inhibitory tag can slow responses if a subsequent go trial (actor's stimulus) is also presented on the right side (compatible). Analogously, if the person on the left responds to a compatible trial in $N-1$ and the stimulus is presented on the left side, again an inhibitory tag is applied for the person on the right side on a nogo trial. In a subsequent go trial, his response is slowed if the stimulus appears on the left side (incompatible). At the same time, responses are considerably faster following nogo trials when the subsequent go stimulus appears in the non-inhibited location (compatible-compatible and incompatible-incompatible trial transitions for $N-1$ to $N$ ).

If this is correct, several conclusions can be drawn. First, inhibitory tags following go/nogo discrimination can mimic the RT pattern of sequential modulation effects that are typically observed in standard Simon tasks. Second, such a pattern should be especially pronounced in nogo/go transitions but not in go/go transitions, which do not contain inhibitory tags. Third, go/nogo discrimination (and its inhibitory consequences) applies to both Individual- and Joint go/nogo tasks alike. Consequently, sequential modulations should be expected to occur for Individual and Joint go/nogo tasks. Furthermore, if sequential modulation effects are found for both task types, one can also predict that Simon-like effects are especially likely to occur after compatible trials in $N-1$. Although Simon-like effects 
are typically not found in Individual go/nogo tasks, this possibility could, however, have remained undetected in previous studies.

Therefore, an analysis of trial-to-trial sequential dependencies might help to gain a better understanding of the processes that take place following compatible and incompatible trials in a Joint and Individual Simon task. This might offer insights into the commonalities and differences between these two conditions. In brief, we expect processing mechanisms to be similar in Individual and Joint go/nogo Simon tasks, and this should be reflected in sequential modulations of Simon-like effects for both task types especially in nogo/go trial transitions. The increased Simon-like effect typically found in the Joint compared to the Individual go/nogo task might be related to stronger inhibitory tags in the social situation and/or to an extended go/nogo discrimination in terms of a self/other discrimination, which thus makes the effect pattern more pronounced.

\section{Method}

\section{Participants}

A group of 24 undergraduate students (12 male; mean age, 24.8 years; $\mathrm{SD}=2.9$ ) participated in this experiment. All were right-handed, had normal or corrected-to-normal vision, and were naive with regard to the hypotheses of the experiment. They were paid $€ 7$ for taking part. Participants gave a written informed consent to participate in the study, which was conducted in accordance with the standards of the ethics committee of the University of Leipzig and the ethical standards laid down in the 1975 Declaration of Helsinki.

\section{Apparatus and stimuli}

Participants were seated in a sound-attenuated dimly lit room. Responses were recorded with two keys placed on a table at a distance of $25 \mathrm{~cm}$ from each other. All stimuli were displayed on a computer monitor in white on a black background at a constant viewing distance of $60 \mathrm{~cm}$. The fixation point in the center of the screen was marked by a plus sign $\left(0.9^{\circ} \times 0.9^{\circ}\right)$. Stimuli consisted of squares and diamonds $\left(1.9^{\circ} \times 1.9^{\circ}\right)$, presented to the left or right of the fixation with an eccentricity of $5.7^{\circ}$ visual angle.

\section{Procedure}

In the Standard Simon control task, participants responded by pressing the left key with the index finger of their left hand for the square and the right key with their right index finger for the diamond. The stimuli randomly appeared on the left or the right of the centrally presented fixation cross. Participants were seated in front of the monitor to either the left or the right side so as to keep the seating position identical in all three tasks with the empty chair remaining in place in the Individual go/nogo task (see Fig. 1).

In the Individual and the Joint go/nogo tasks, participants responded to one of the shapes only (e.g., squares) by making a simple discrimination response. They were asked to refrain from responding if the other shape (e.g., diamond) appeared. In the Joint go/nogo task, they performed the identical task sitting alongside another person who responded to the other stimulus.

Each trial began with the presentation of a fixation cross for $250 \mathrm{~ms}$. The target stimulus (square or diamond) appeared together with the fixation cross for $150 \mathrm{~ms}$. Responses had to be given within 1,800 ms. In the case of correct responses, the fixation cross was provided as feedback for $300 \mathrm{~ms}$. If no response was given within
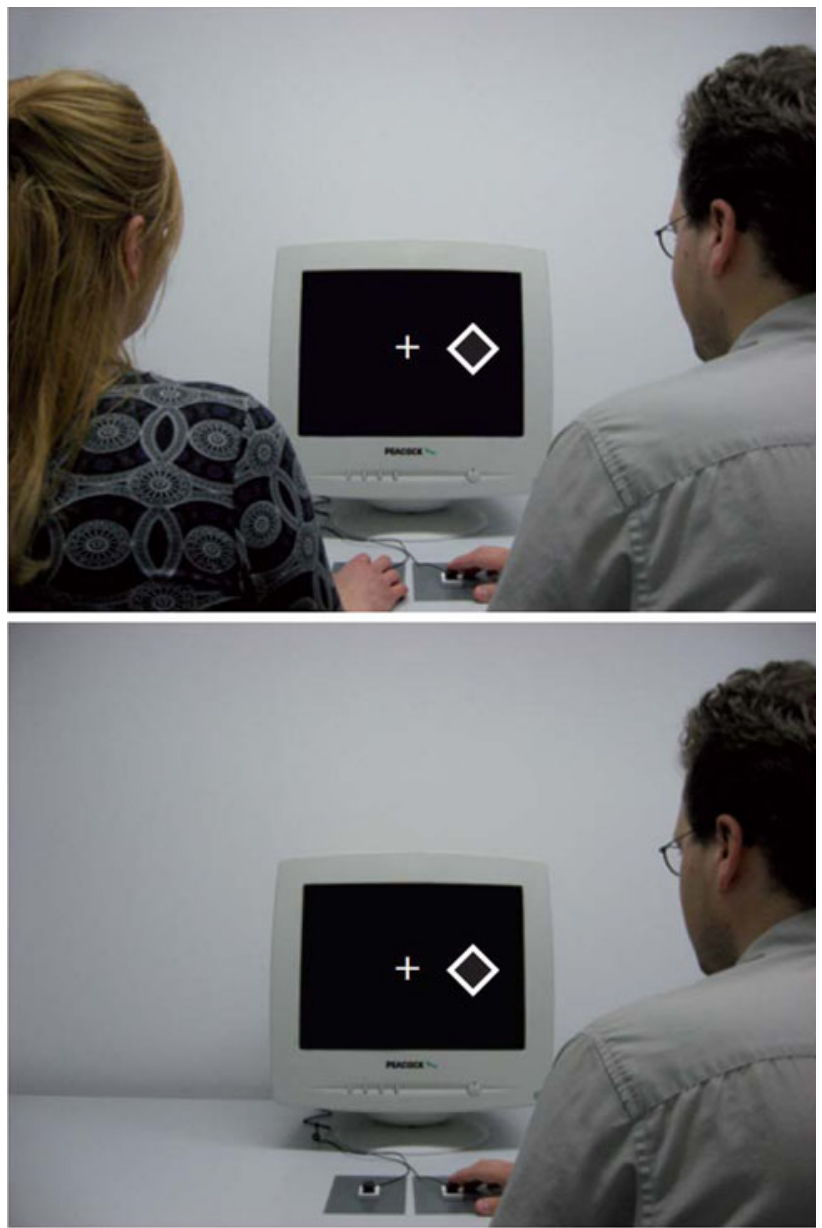

Fig. 1 Experimental setting in the Joint go/nogo task (upper panel) and Individual go/nogo task (lower panel) 
$1,800 \mathrm{~ms}$ after stimulus onset, the feedback "zu langsam" (too slow) was shown. In the case of an incorrect response, error feedback "Fehler" (error) was provided. All forms of feedback (fixation cross, too slow, or error) were displayed for $300 \mathrm{~ms}$. Following feedback, there was a constant inter-trial interval of $1,750 \mathrm{~ms}$ before the next trial started. In each task, participants completed five experimental blocks of 112 trials, separated by short breaks. Before each task, participants performed a block of 66 practice trials. The order of tasks was counterbalanced across pairs of participants. Half of the participants started with the Individual go/nogo task, followed by the Joint go/nogo task, and performed the Standard Simon control task at the end. The other half of participants started with the Joint go/nogo task, followed by the Individual go/nogo task, and performed the Standard Simon control task at the end.

RTs in the two go/nogo tasks were analyzed as a function of setting (Individual vs. Joint), transition (go/ go, nogo/go, for $N-1$ to $N$, respectively), compatibility between stimulus location and response location in trial $N-1$ (compatible, incompatible), and compatibility in trial $N$ (compatible, incompatible). Figure 2 illustrates the different possible trial transitions in the Joint go/nogo task for the person(s) responding in trial $N-1$ and trial $N$. With respect to the transition analyses, we cannot directly compare the Standard Simon task with the other two go/nogo tasks, because a standard Simon task does not contain real nogos. We therefore performed an additional analysis in which we compared Simon effects for all three tasks with respect to their distributional trends.

\section{Results}

Data analysis

Prior to statistical analyses, all trials in which responses were incorrect on either the current $(2.8 \%)$ or previous trial $(2.9 \%)$, faster than $150 \mathrm{~ms}$ or slower than $1,000 \mathrm{~ms}$ $(<0.1 \%)$ were excluded from statistical RT analyses. The first trial in each block was also eliminated prior to analysis. Error rates were rather low over all tasks, with $4.4 \%$ in the Standard Simon task, $2.2 \%$ in the Joint go/nogo task, and $0 \%$ in the Individual go/nogo task. This reflects the ease of a simple stimulus discrimination task. Because of the low number of overall errors, errors rates were not analyzed further.

The Standard Simon task showed the following effects. We found a significant main effect of Compatibility, $F(1$, $23)=39.41, \mathrm{MSe}=793.33, p<0.001$, partial $\eta^{2}=0.63$, showing an overall Simon effect with faster responses in S-R compatible trials (426 ms) than in incompatible trials (452 ms). We also found the typical sequential modulation of the Simon effect revealed by a significant interaction of Preceding Compatibility $\times$ Compatibility, $F(1,23)=$ 127.67, $\mathrm{MSe}=264.11, \quad p<0.001$, partial $\eta^{2}=0.85$, showing a larger Simon effect after compatible trials (52 $\mathrm{ms})$ than after incompatible trials $(-1 \mathrm{~ms})$.
Fig. 2 Hypothetical trial transitions from trial $N-1$ to trial $N$, separately for go/go and nogo/go transitions in a Joint go/nogo task. In the given example, the person on the right responds to the diamond and the person on the left to the square. Compatibility ( $C$ compatible, $I C$ incompatible) is always coded for the person responding (shown in blue) (color figure online)

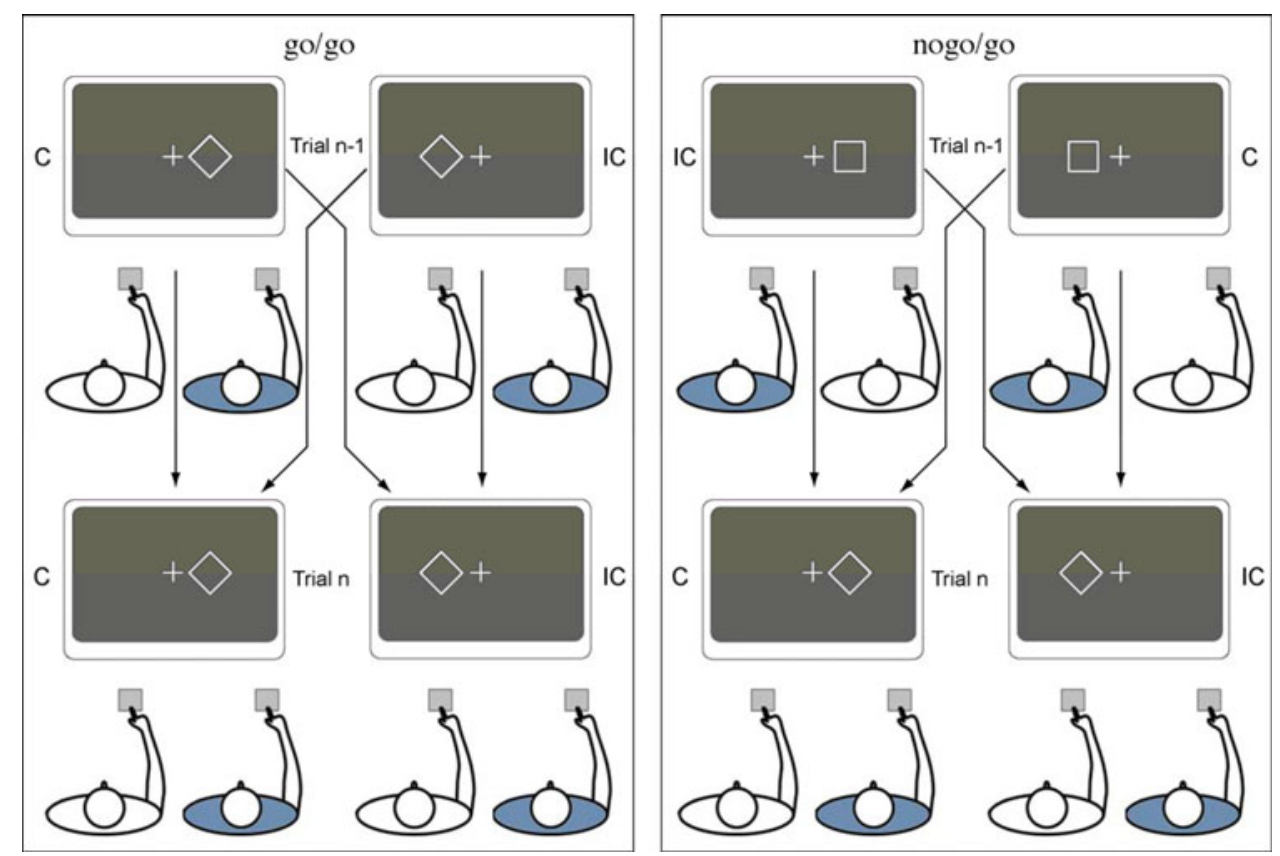


The social Simon effect

To investigate the social Simon effect, mean RTs were computed as a function of Setting (Individual go/nogo vs. Joint go/nogo), Transition (go vs. nogo in trial ${ }_{N-1}$ ), Preceding Compatibility $_{N-1}$ (compatible vs. incompatible) and Compatibility $_{N}$ (compatible vs. incompatible). The comparison with the Standard Simon task was performed in a separate analysis (see below) excluding the transition factor because nogo transitions reflect response switches in the Standard Simon task and not a valid nogo, as in the other two tasks.

We found a significant main effect of Compatibility, $F(1,23)=9.34, \mathrm{MSe}=337, p<0.05$, partial $\eta^{2}=0.29$, which indicated that irrespective of setting, responses were faster with S-R compatibility (362 ms) than with incompatibility (368 ms), reflecting an overall go/nogo Simon effect. Responses were also faster in the Joint go/nogo (349 $\mathrm{ms}$ ) compared to the Individual go/nogo task (381 ms), suggesting a social facilitation effect as confirmed by the main effect of Setting, $F(1,23)=45.86$, $\mathrm{MSe}=2140.0, p<0.001$, partial $\eta^{2}=0.66$. The factors Setting $\times$ Compatibility interacted, $F(1,23)=4.64$, $\mathrm{MSe}=208.26, p<0.05$, partial $\eta^{2}=0.17$. Follow-up analyses confirmed a significant Simon effect of $9 \mathrm{~ms}$ in the Joint go/nogo task, $t(23)=-3.93, p \leq 0.001$, while the $3 \mathrm{~ms}$ effect in the Individual go/nogo tasks was not significant, $t(23)>0.05$. The main effect of Transition was also significant, $F(1,23)=18.21, \quad \mathrm{MSe}=696.49$, $p<0.001$, partial $\eta^{2}=0.44$, showing faster responses after go trials $(360 \mathrm{~ms})$ than after nogo trials $(371 \mathrm{~ms})$.

Sequential modulation effects

Reaction times also depended on compatibility in trial $N-1$. First, responses were slightly faster with $\mathrm{S}-\mathrm{R}$ compatibility (364 ms) than incompatibility (366 ms) in the preceding trial, $F(1,23)=5.44, \quad \mathrm{MSe}=88.57$, $p<0.05$, partial $\eta^{2}=0.19$. Furthermore, we also observed a significant interaction of Preceding Compatibility $\times$ Compatibility, $F(1,23)=173.50, \quad \mathrm{MSe}=147.54$, $p<0.001$, partial $\eta^{2}=0.88$, showing a larger Simon effect after compatible trials than after incompatible trials, reflecting a typical sequential modulation of the Simon effect. This sequential modulation was more pronounced in the Joint go/nogo task than in the Individual go/nogo task (see Fig. 3; Table 1), as suggested by the significant threeway interaction of Setting $\times$ Preceding Compatibility $\times$ Compatibility, $F(1,23)=9.08, \quad \mathrm{MSe}=113.12$, $p<0.05$, partial $\eta^{2}=0.28$.

For the Joint go/nogo task, planned comparisons showed a large Simon effect after Simon compatible trials (29 ms), $t(23)=-9.01, p<0.001$, and an inverse Simon effect after Simon incompatible trials $(-11 \mathrm{~ms}), t(23)=4.53$, $p<0.001$. Importantly, a similar pattern was found for the Individual go/nogo task. Again, a significant Simon effect was obtained after Simon compatible trials (16 ms), $t(23)=-5.04, p<0.001$, but was reversed after Simon incompatible trials $(-10 \mathrm{~ms}), t(23)=3.54, p<0.05$ (see Fig. 3). We found evidence of a sequential modulation in both the Individual and the Joint go/nogo tasks. We additionally compared the size of the sequential modulation for trials following Simon compatible and Simon incompatible trials. The $29 \mathrm{~ms}$ effect following Simon compatible trials in the Joint go/nogo task was significantly larger than the $16 \mathrm{~ms}$ effect in the Individual go/nogo task, $F(1$, $23)=10.37, \mathrm{MSe}=94.76, p<0.05$, partial $\eta^{2}=0.31$. Following Simon incompatible trials, the reversed Simon effect for the Joint $(-11 \mathrm{~ms})$ and the Individual go/nogo task $(-10 \mathrm{~ms})$ did not differ $(F<1)$.

\section{Transition effects}

The sequential modulation was stronger for nogo/go transitions than for go/go transitions, as indicated by a significant interaction of Transition $\times$ Preceding
Fig. 3 Simon effect in trial $N$, depending on compatibility ( $C$ compatible, $I C$ incompatible) of the previous trials (sequential modulation) for the Individual go/nogo task (left panel), the Joint go/nogo task (middle panel) and the Standard Simon task (right panel), respectively
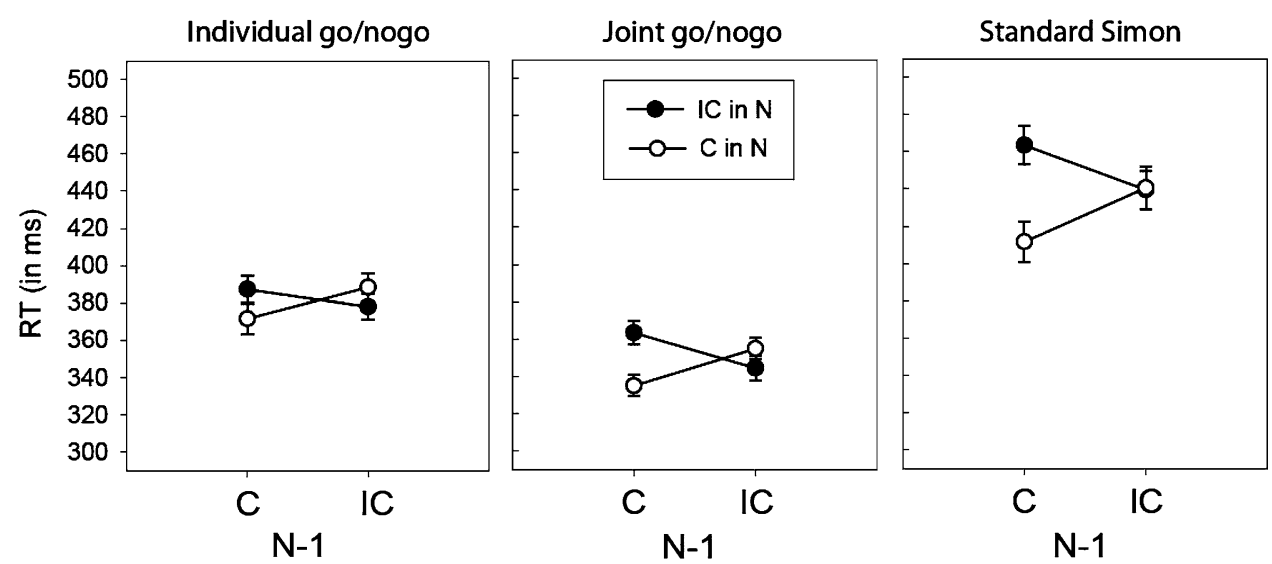
Table 1 Mean reaction times (RTs) in milliseconds obtained for all three settings (Individual go/nogo, Joint go/nogo and Standard Simon), trial transitions (go/go and nogo/go), preceding compatibility in trial $N-1\left(\mathrm{C}_{-} N-1\right.$ : compatible and $\mathrm{IC}_{-} N-1$ : incompatible) and Simon compatibility in trial $\mathrm{N}$ (C_N $N$ : compatible and $\mathrm{IC} \_N$ : incompatible)

\begin{tabular}{|c|c|c|c|}
\hline \multicolumn{2}{|c|}{ Transition (go/go) } & \multicolumn{2}{|c|}{ Transition (nogo/go) } \\
\hline$C_{-} N-1$ & $I C_{-} N-1$ & $C_{-} N-1$ & $I C_{-} N-1$ \\
\hline IC_N $N$ & C__ $N \quad$ IC_ $\_~$ & C_N $N$ IC_ $N$ & C__ $N$ IC $\_N$ \\
\hline
\end{tabular}

Task

\begin{tabular}{lllllllll} 
Individual & 368 & 374 & 378 & 382 & 375 & 400 & 400 & 374 \\
Joint & 337 & 353 & 343 & 342 & 333 & 373 & 367 & 346 \\
Standard & 417 & 470 & 446 & 444 & 406 & 457 & 435 & 435 \\
\hline
\end{tabular}

Compatibility $\times$ Compatibility, $\quad F(1, \quad 23)=42.39$, $\mathrm{MSe}=307.17, p<0.001$, partial $\eta^{2}=0.65$. This effect was independent of the setting in which participants performed the task (Individual vs. Joint go/nogo task), as the four factors did not interact, $F<1$. Finally, a significant three-way interaction of Setting $\times$ Transition $\times$ Preceding Compatibility, $F(1,23)=8.82, \mathrm{MSe}=176.86, p<0.05$, partial $\eta^{2}=0.28$, indicated a stronger dependence on preceding compatibility for go/go trials in the Individual go/nogo task than in the Joint go/nogo task. No further effects reached the level of significance (all $p \mathrm{~s}>0.05$ ).

To check for a potential task order effect as an explanation of the observed sequential modulation effects found in the Individual go/nogo task, we performed an additional ANOVA in which we added Task Order (Individual-Joint, Joint-Individual) to the given analyses. Task Order did not interact with Setting $\times$ Preceding Compatibility $\times$ Compatibility $(F<1)$, nor with Setting $\times$ Transition $\times$ Preceding Compatibility $\times$ Compatibility $(F<1)$, indicating that task order cannot explain the finding of a sequential modulation in the Individual go/nogo task.

\section{Comparing Individual, Joint and Standard Simon tasks}

There are several methods to analyze distributional trends. A standard Simon task typically shows an increased Simon effect with generally faster RTs (Hommel, 1993). We performed RT distribution analyses including all three tasks by dividing RTs in quartiles (bins) from the fastest to the slowest (see De Jong et al., 1994; but see also Burle, Possamaï, Vidal, Bonnet, \& Hasbroucq, 2002; de Jong et al., 1994; Ridderinkhof, van den Wildenberg, Wijnen, \& Burle, 2004). The difference between the averaged RTs for related bins of the distributions then represents the average size of the Simon effect for the specific time-window spanned by these bins. To compare distributional trends in all three tasks, we conducted an ANOVA including the factors Setting (Individual, Joint, Standard Simon),
Compatibility $_{N}$ (compatible vs. incompatible) and Quartile, with all factors as within-subject variables.

This analysis showed a significant three-way interaction of Setting $\times$ Compatibility $\times$ Quartile, $F(6,138)=3.72$, MSe $=279.66, \quad p<0.05, \quad$ partial $\eta^{2}=0.14$. While response speed neither affected the size of the Simon effect in the Individual go/nogo task $(F<1)$ nor in the social Simon task $(F<1)$, we did find the typical RT distribution effect in the Standard Simon task, $F(3,69)=6.90$, $\mathrm{MSe}=276.86, \quad p<0.05$, partial $\eta^{2}=0.23$, indicating increased Simon effects with shorter RTs. The distributional trends did not differ between the Individual and Joint go/nogo tasks $(F<1)$, but we found a significant difference between the Joint go/nogo task and the Standard Simon task, $F(3,69)=4.95$, MSe $=181.99, p<0.05$, partial $\eta^{2}=0.18$ (see Fig. 4 ).

In order to test if the narrower RT range in the Joint go/ nogo tasks as compared to the Standard Simon task might account for the different pattern of effects, we additionally analyzed distributional trends only for overlapping RT ranges. This was done by comparing distributional trends of Quartile two (334 ms), three (372 ms) and four (455 ms) in the Joint go/nogo task with Quartile one $(328 \mathrm{~ms})$, two (394 ms) and three (453 ms) in the Standard Simon task. We again obtained the finding of a significant three-way interaction of Setting $\times$ Compatibility $\times$ Quartile, $F(2,46)=$ 10.35, MSe $=105.82, p<0.05$, partial $\eta^{2}=0.31$, ruling out the possibility that the variability in RTs between conditions can account for the differences in distributional trends between the Joint go/nogo and the Standard Simon task.

\section{Discussion}

The present study was designed to investigate the underlying mechanisms of the social Simon effect in more detail. In particular, we studied the role of low-level trialto-trial feature transition effects that typically lead to sequential modulations of the Simon effect in a standard Simon task. In a social Simon task, however, such trial-totrial transitions not only contain $\mathrm{S}-\mathrm{R}$ features but also information about the go/nogo character of the preceding trial (e.g., tags not to respond) and might thus reflect another quality than in a standard Simon task. We compared the performance in an Individual go/nogo task, in which participants performed the task alone, with that in a Joint go/nogo task, in which a participant performed the task with another person.

Using non-social stimuli, we were able to replicate previous findings (Sebanz et al., 2003; Tsai et al., 2006) showing an overall Simon effect in the Joint go/nogo task, but not in the Individual go/nogo task, which is in line with 
Fig. 4 Distributional analysis of reaction time (RT) data divided into quartiles, that is, bins of equal proportion, depending on compatibility ( $C$ compatible, $I C$ incompatible) in trial $N$ for the Individual go/ nogo task (left panel), the Joint go/nogo task (middle panel) and the Standard Simon task (right panel)
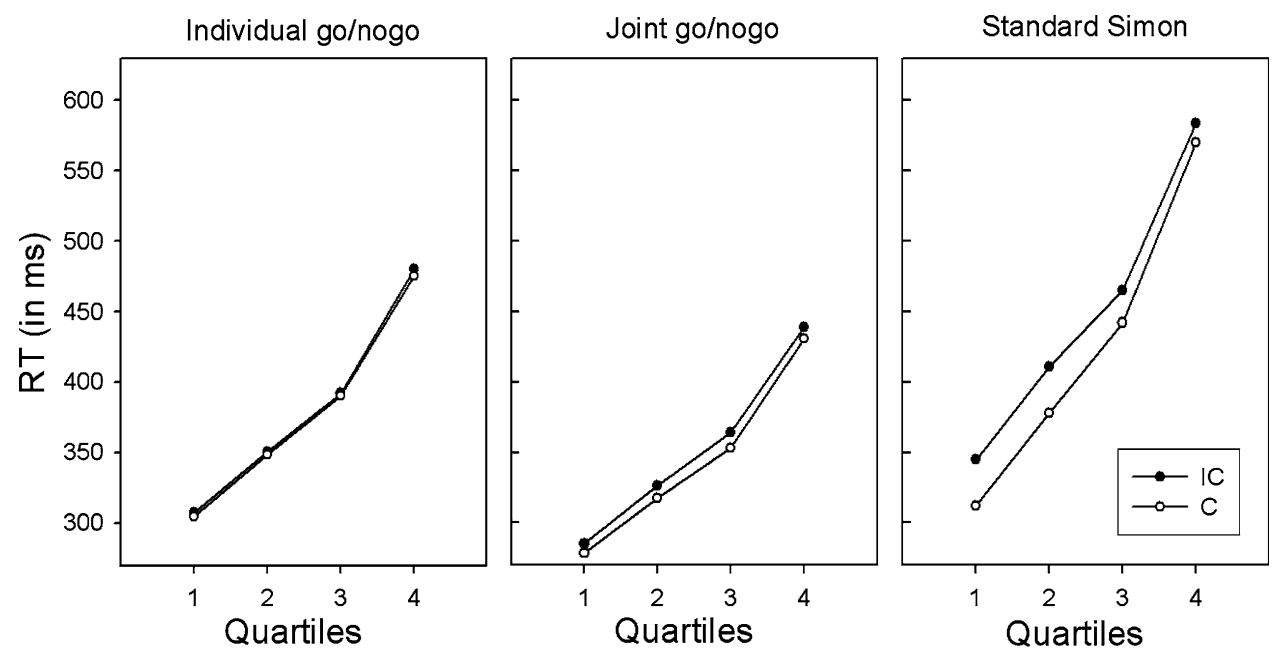

the assumption of action co-representation. Taking trial-totrial transitions into account, in the Joint and, importantly, also in the Individual go/nogo task, we found a sequential modulation of the Simon effect that mimics the pattern of sequential modulation effects typically found in standard Simon tasks. As expected, the sequential modulation was generally stronger for nogo/go transitions than for go/go transitions, probably reflecting the need to inhibit responses in nogo trials. Importantly, this nogo effect occurred irrespective of task type (i.e., Individual and Joint go/nogo tasks). These findings suggest the involvement of an inhibitory response tag process (Neill, Valdes, Terry, \& Gorfein, 1992) in the Joint as well as in the Individual go/nogo task, which implies that similar processing mechanisms are involved in both task types. In nogo trials, for example, inhibitory response tags with respect to position information seem to be attached to either the stimulus or response code. When the person's own stimulus (go trial) subsequently appears (in trial $\mathrm{N}$ ) on the side that is now attached with an inhibitory response tag, responses are slowed.

In the Individual go/nogo task, the reversed Simon effect following Simon incompatible trials and the positive Simon effect following Simon compatible trials were of similar magnitude. This was not the case for the Joint go/ nogo task, in which the Simon effect following compatible trials was larger than the reversed effect following incompatible trials, a finding which we will subsequently discuss in depth.

The finding of a sequential modulation in the Individual go/nogo task cannot be explained by task order assumptions (e.g., because the Joint go/nogo task is performed prior to the Individual go/nogo task). First, we counterbalanced for task order. Second, analyses including task order as a factor showed no interactions between the sequence effects in the Individual and Joint go/nogo tasks and order, both when including and excluding the transition factor. These findings suggest that the sequential modulation of the Simon effect in the Individual go/nogo task is not due to transfer effects from the Joint to the Individual go/nogo task.

The comparison of the distributional trends across all three tasks showed the typical RT distribution effect, that is, increased Simon effects with smaller RTs (Hommel, 1993), only in the Standard Simon task. However, response speed did not affect the size of the Simon effect in the Individual go/nogo task, nor in the Joint go/nogo task. Consequently, while the RT distributions did not differ for the latter two tasks, we found a significant difference in the distributional trends between the Joint go/nogo and the Standard Simon task. The similarity in distributional patterns between the Joint and the Individual go/nogo tasks further substantiates the assumption of common processes involved in the Joint and the Individual go/nogo tasks.

So far, we have shown that the Individual go/nogo task and the Joint go/nogo task are rather similar in their processing characteristics. For both task types, we found trialto-trial sequential modulations, reliable Simon-like effects (at least following compatible trials), no task type specific differences for go/go and nogo/go transitions, and a similar lack of sensitivity in RT distributions (in contrast to the Standard Simon task). Therefore, we conclude that Individual and Joint go/nogo tasks might rely on similar lowlevel (e.g., inhibitory) mechanisms.

Despite this similarity, however, the differences in RT patterns (e.g., Simon-like effect after compatible trials in $N-1$ and the sequential modulation) were more pronounced in the Joint than in the Individual go/nogo task. These findings are in line with the conventional action corepresentation account that might work on top of the lowlevel trial-to-trial transition effects. Alternatively, one could speculate that inhibitory tags might be stronger (e.g., more effective) when a task is shared with another person 
(Joint go/nogo task) compared to the Individual go/nogo task. One potential reason for this might be that in the Joint go/nogo task, an inhibitory process in nogo trials may be related to the other person so that a go/nogo decision may now be resolved by (additional) self-other discrimination (Philipp \& Prinz, 2010). When another person is present, as in the Joint go/nogo task, the simple go/nogo discrimination may be extended to the level of between-person discrimination (e.g., me vs. other; my space vs. the other's space; see Hommel, Colzato, \& van den Wildenberg, 2009). An agent-compatible stimulus position (with respect to the actor's response position) encourages the use of the stimulus position for determining whose turn it is. This assumption is in line with recent findings showing that the social Simon effect is diminished when two participants performed the task outside of each other's peripersonal space (Guagnano, Rusconi, \& Umiltà, 2010). Taking this view, a compatible stimulus position (with respect to both actors' response positions) may automatically prime whether it is "my" or the other person's turn (self-other discrimination).

Taken together, the present findings suggest that lowlevel feature binding mechanisms related to the requirement not to respond on nogo trials contribute to the social Simon effect. Further research is clearly needed to elaborate to which extent the proposed inhibitory mechanisms might also account for the social Simon effect in general.

\section{References}

Akçay, Ç., \& Hazeltine, E. (2007). Conflict monitoring and feature overlap: Two sources of sequential modulations. Psychonomic Bulletin \& Review, 14, 742-748.

Ansorge, U., \& Wühr, P. (2004). A response-discrimination account of the Simon effect. Journal of Experimental Psychology: Human Perception and Performance, 30, 365-377.

Botvinick, M. M., Braver, T. S., Barch, D. M., Carter, C. S., \& Cohen, J. D. (2001). Conflict monitoring and cognitive control. Psychological Review, 108, 624-652.

Burle, B., Possamaï, C.-A., Vidal, F., Bonnet, M., \& Hasbroucq, T. (2002). Executive control in the Simon effect: An electromyographic and distributional analysis. Psychological Research, 66, 324-339.

De Jong, R., Liang, C.-C., \& Lauber, E. (1994). Conditional and unconditional automaticity: A dual-process model of effects of spatial stimulus-response correspondence. Journal of Experimental Psychology: Human Perception and Performance, 20, 731-750.

Fischer, R., Dreisbach, G., \& Goschke, T. (2008). Context-sensitive adjustments of cognitive control: Conflict-adaptation effects are modulated by processing demands of the ongoing task. Journal of Experimental Psychology: Learning, Memory, and Cognition, 34, 712-718.

Gratton, G., Coles, M. G. H., \& Donchin, E. (1992). Optimizing the use of information: Strategie control of activation of responses. Journal of Experimental Psychology: General, 121, 480-506.
Guagnano, D., Rusconi, E., \& Umiltà, C. A. (2010). Sharing a task or sharing space? On the effect of the confederate in action coding in a detection task. Cognition, 114, 348-355.

Hommel, B. (1993). The relationship between stimulus processing and response selection in the Simon task: Evidence for a temporal overlap. Psychological Research, 55, 280-290.

Hommel, B. (1998). Event files: Evidence for automatic integration of stimulus-response episodes. Visual Cognition, 5, 183-216.

Hommel, B., Colzato, L. S., \& van den Wildenberg, W. P. M. (2009). How social are task representations? Psychological Science, 20, 794-798.

Hommel, B., Müsseler, J., Aschersleben, G., \& Prinz, W. (2001). The theory of event coding (TEC): A framework for perception and action planning. Behavioral and Brain Sciences, 24, 849-878.

Hommel, B., Proctor, R. W., \& Vu, K.-P. L. (2004). A featureintegration account of sequential effects in the Simon task. Psychological Research, 68, 1-17.

Liepelt, R., von Cramon, D. Y., \& Brass, M. (2008). What is matched in direct matching? Intention attribution modulates motor priming effects. Journal of Experimental Psychology: Human Perception and Performance, 34, 578-591.

Lu, C.-H., \& Proctor, R. W. (1995). The influence of irrelevant location information on performance: A review of the Simon and spatial Stroop effects. Psychonomic Bulletin \& Review, 2, 174-207.

Neill, W. T., Valdes, L. A., Terry, K. M., \& Gorfein, D. S. (1992). Persistence of negative priming. II. Evidence for episodic trace retrieval. Journal of Experimental Psychology: Learning, Memory, and Cognition, 18, 993-1000.

Newman-Norlund, R., van Schie, H. T., van Zuijlen, A., \& Bekkering, H. (2007). The mirror neuron system is more active during complementary compared with imitative action. Nature Neuroscience, 10, 817-818.

Nieuwenhuis, S., Stins, J. F., Posthuma, D., Polderman, T. J. C., Boomsma, D. I., \& De Geus, E. J. (2006). Accounting for sequential trial effects in the flanker task: Conflict adaptation or associative priming? Memory \& Cognition, 34, $1260-1272$.

Notebaert, W., Soetens, E., \& Melis, A. (2001). Sequential analysis of a Simon task-evidence for an attention-shift account. Psychological Research, 65, 170-184.

Philipp, A. M., \& Prinz, W. (2010). Evidence for a role of the responding agent in the joint compatibility effect. Quarterly Journal of Experimental Psychology, 28, 1-13.

Prinz, W. (1997). Perception and action planning. European Journal of Cognitive Psychology, 9(2), 129-154.

Ramnani, N., \& Miall, R. C. (2004). A system in the human brain for predicting the actions of others. Nature Neuroscience, 7, 85-90.

Ridderinkhof, K. R., van den Wildenberg, W. P. M., Wijnen, J., \& Burle, B. (2004). Response inhibition in conflict tasks is revealed in delta plots. In M. Posner (Ed.), Cognitive Neuroscience of Attention. New York: Guilford Press.

Sebanz, N., Bekkering, H., \& Knoblich, G. (2006). Joint actions: Bodies and minds moving together. Trends in Cognitive Sciences, 10, 70-76.

Sebanz, N., Knoblich, G., \& Prinz, W. (2003). Representing others' actions: Just like one's own? Cognition, 88, B11-B21.

Sebanz, N., Knoblich, G., \& Prinz, W. (2005). How two share a task: Corepresenting stimulus-response mappings. Journal of Experimental Psychology: Human Perception and Performance, 31, 1234-1246.

Simon, J. R. (1990). The effects of an irrelevant directional cue on human information processing. In R. W. Proctor \& T. G. Reeve (Eds.), Stimulus-response compatibility: An integrated perspective. Advances in Psychology (vol. 65, pp. 31-86) Amsterdam: North-Holland. 
Simon, J. R., Hinrichs, J. V., \& Craft, J. L. (1970). Auditory S-R compatibility: Reaction time as a function of ear-hand correspondence and ear-response-location correspondence. Journal of Experimental Psychology, 86, 97-102.

Simon, J. R., \& Rudell, A. P. (1967). Auditory S-R compatibility: The effect of an irrelevant cue on information processing. Journal of Applied Psychology, 51, 300-304.

Stürmer, B., Leuthold, H., Soetens, E., Schröter, H., \& Sommer, W. (2002). Control over location-based response activation in the Simon Task: Behavioral and Electrophysiological evidence. Journal of Experimental Psychology: Human Perception and Performance, 28, 1345-1363.

Tsai, C. C., \& Brass, M. (2007). Does the human motor system simulate Pinocchio's actions? Co-acting with a human hand versus a wooden hand in a dyadic interaction. Psychological Science, 18, 1058-1062.

Tsai, C.-C., Kuo, W. J., Jing, J. T., Hung, D. L., \& Tzeng, O. J. L. (2006). The common coding framework in self-other interaction: Evidence from joint action task. Experimental Brain Research, $175,353-362$. van Schie, H. T., Mars, R. B., Coles, M. G. H., \& Bekkering, H. (2004). Modulation of activity in medial frontal and motor cortices during error observation. Nature Neuroscience, 7, 549-554.

Wallace, R. A. (1971). S-R compatibility and the idea of a response code. Journal of Experimental Psychology, 88, 354-360.

Wendt, M., Kluwe, R. H., \& Peters, A. (2006). Sequential modulations of interference evoked by processing task-irrelevant stimulus features. Journal of Experimental Psychology: Human Perception and Performance, 32, 644-667.

Winkel, J., Wijnen, J. G., Ridderinkhof, K. R., Groen, I., Derrfuss, J., Danielmeier, C., et al. (2009). Your conflict matters to me! Behavioral and neural manifestations of control adjustments after self-experienced and observed decision-conflict. Frontiers in Human Neuroscience, 3, 1-8.

Wühr, P. (2005). Evidence for gating of direct response activation in the Simon task. Psychonomic Bulletin \& Review, 12, 282-288.

Wühr, P., \& Ansorge, U. (2005). Exploring trial-by-trial modulations of the Simon effect. Quaterly Journal of Experimental Psychology A, 58, 705-731. 